\title{
Enfermedad, hundimiento, derrota. La caída de Albert Camus
}

\author{
Illness, Collapse, Defeat. Albert Camus' The Fall
}

\author{
DAVID MONTERO BOSCH*
}

\begin{abstract}
Resumen: La enfermedad fue un acontecimiento importante en la vida de Albert Camus, que fue marcado por la tuberculosis desde su juventud y se vio obligado a luchar contra ella hasta su muerte. A pesar de ello, los efectos de la enfermedad en sí misma en la obra literaria de Camus no han sido estudiados como se podría esperar. Este texto está centrado en La Caída (La Chute), una pequeña novela donde la enfermedad tiene un fuerte contenido simbólico que abarca el terreno de la antropología, la moral y la política.

Palabras clave: Camus, enfermedad, vergüenza, orgullo, confesión, nihilismo.
\end{abstract}

\begin{abstract}
Illness was a major event in the life of Albert Camus. He was marked by tuberculosis since his youth and he had to fight the disease till his death. Despite this fact, the effects of the illness in Camus' literary work have scarcely been studied. This paper is focused on The Fall ( $\mathrm{La}$ Chute), a little novel where the disease has a strong symbolic sense that covers anthropological, moral and political fields.
\end{abstract}

Keywords: Camus, illness, shame, pride, confession, nihilism.

\section{El miedo del guardameta ante el penalti ${ }^{1}$}

Cuando el delantero coloca el balón en el punto de penalti, el guardameta sabe que lo que va a ocurrir a continuación se escapa de sus manos y pertenece al mundo del destino o del azar. Si no es miedo es algo parecido lo que siente en la fracción de segundo en la que el delantero inicia la carrerilla para golpear la pelota.

Algo de esto debió experimentar en ocasiones Albert Camus cuando jugaba como portero en el equipo junior del Racing universitario de Alger. Y algo semejante, pero mucho más intenso, debió sentir cuando en 1930, con 17 años de edad, comienza a escupir sangre. "Pañuelos empapados, dedos llenos de sangre: a veces tiene vértigo. Escupe sangre, escupe

Fecha de recepción: 21/05/2016. Fecha de aceptación: 12/09/2016.

* Universidad de Valencia. damontero22@hotmail.es

1 Die Angst des Tormanns beim Elfmeter, novela de Peter Handke (1970) y película de Wim Wenders (1972). 
su propia vida", dice Oliver Todd ${ }^{2}$. En aquellos años, padecer la tuberculosis en un barrio pobre de Alger era prácticamente fatal y, en todo caso, entrar en una pendiente sin futuro: paro, imposibilidad de emigrar a Francia... en uno de cada tres pacientes, la muerte.

Puede decirse que, en el caso de Camus, fue la irrupción del absurdo en su vida de manera súbita. Años más tarde, en El mito de Sísifo, describirá el absurdo como la extrañeidad (étrangeté) del mundo ${ }^{3}$. El ser humano siente un ansia inextinguible de unidad con el mundo. Quiere que sus deseos se realicen, que su conocimiento abarque la totalidad, que sus ansias de empatía se conviertan en solidaridad universal y el bien sea ley. En vez de eso, el mundo le presenta un muro contra el que chocan deseos, saber y justicia. Para Camus, antes de ponerle nombre, un elemento fundamental de ese muro fue la enfermedad. No sólo como amenaza de muerte, el horizonte definitivo, la anulación del sentido del vivir, sino como impedimento permanente, obstáculo insoslayable, límite de la libertad. En primer lugar, debe abandonar sus actividades deportivas, que fue algo menos banal de lo que se puede suponer -el fútbol fue una pasión toda su vida, una pasión psicológica y moral-. Más tarde, un informe negativo del inspector médico le impide el acceso a la enseñanza de la filosofía. Irrupciones constantes de la enfermedad le acosarán siempre. En las anotaciones de sus viajes, lo más parecido a un diario que conservamos de Camus, hay una referencia constante a los accesos de fiebre, las dificultades para respirar y el abatimiento físico que le tumban en la cama y lo embotan en los momentos menos oportunos. En sus Carnets escribe de la enfermedad: "No soy yo el que renuncia a los seres y a las cosas (no podría) son las cosas y los seres los que renuncian a mî"4. Es la perfecta expresión de la extrañeidad del mundo.

En esas circunstancias, es obvio que la enfermedad debería ocupar un lugar en la obra literaria de Camus y así ocurre. Ya hace acto de presencia en su primera novela, no publicada, La muerte feliz (1936-38). En forma de peste, es el tema central de la novela de este título (La peste, 1947) y en Estado de sitio (1948), pieza teatral en muchos sentidos gemela de la anterior. En las dos se juega con una doble simbología. La enfermedad, la plaga, se refiere a la muerte como destino existencia, por un lado, y al totalitarismo como sistema político aniquilador de lo vital, por otro. Sin embargo, en otras obras, la enfermedad juega con otros significados, emparentados, pero diferentes de los mencionados. En especial, en su última novela acabada y publicada en vida, la intrigante La caída (La Chute, 1956). Mi objetivo es analizar el impacto de la enfermedad en el pensamiento de Camus a través de esta obra.

\section{La caída, una obra simbólica}

La caída desconcierta a sus críticos en el primer momento. Acostumbrados a clasificar las obras de Camus de acuerdo con un significado primario, la opacidad de esta pequeña novela les descoloca porque se abre a una multitud de interpretaciones. Las intenciones manifiestas son bastante claras. La novela es una secuela del ácido enfrentamiento que

2 Todd, Oliver: Albert Camus. Una vida, Barcelona, Tusquets, 1997, p. 50.

3 Le mythe de Sisyphe, en Camus, Albert: Euvres complètes, R. Gay-Crosier y J. Lévi-Valensi (eds.), ,4 vol. Col. Bibliothèque de la Pléiade, Paris, Gallimard, 2006, p. 229. A partir de ahora citaré como OC y el $n^{\circ}$ del volumen y página. Las traducciones son personales, salvo indicación contraria.

4 Carnets, OC II, p. 966. 
mantuvo con Jean-Paul Sartre en 1952 a propósito de su libro El hombre rebelde (1951). Sartre le había acusado entonces, entre otras cosas, de incapacidad para la conceptualización filosófica de sus teorías ${ }^{5}$. Camus estuvo dudando durante mucho tiempo si debía responder a las críticas en términos teóricos y no lo hizo. Guardó en su cajón algún trabajo al respecto ${ }^{6}$ y esperó hasta 1956 para dar una réplica en su propio terreno, el literario ${ }^{7}$. El resultado es el retrato del nihilista, de la manera en que Camus entiende el término, que es, para decirlo de manera lapidaria, el que niega la vida. En este caso, la figura del principal representante de los que él llama "nuestros existencialistas", a los que acusa de erigirse en profetas de un futuro de sumisión a los poderes totalitarios. No se trata de una respuesta en el ámbito ideológico, sino, propiamente hablando, un retrato a través de una imaginaria confesión del personaje.

Pero, y sobre todo esto parece haber unanimidad, el problema reside en la forma de la obra, cargada de dobles o triples sentidos. Camus decía que todas sus obras eran simbólicas, pero en ésta no hay una página en la que no se utilice deliberadamente al menos un símbolo. Generalmente son inversiones de los cristianos. Lo es la misma elección de Amsterdam, en donde ocurre la trama de la novela, como ciudad muerta, encerrada en anillos concéntricos de canales, que remiten a los círculos infernales de La Divina Comedia. El seudónimo del protagonista, Jean-Baptiste Clamence, juega con el lado profético del personaje puesto que él también clama en el desierto o eso dice. El agua glauca de los canales y el mar inmóvil que rodea la ciudad sugieren, en palabras de Clamence, una inmensa pila de agua bendita que, en lugar de dar vida, aniquila todo impulso vital. Puestos en esta vía, los críticos pueden enredarse con facilidad en una maraña de significaciones irónicas, metáforas y juegos verbales. Trataré de liberarme de esta tarea, a veces fatigosa, restringiéndome a la interpretación de la enfermedad, que pienso que es el punto fundamental para la interpretación del texto.

Antes, dos palabras sobre el desarrollo y asunto de la narración.

\section{Voluntad de dominación}

La novela es un largo monólogo de un personaje marginal en cinco jornadas. Arranca en un bar de los bajos fondos en el que Clamence aborda a un silencioso interlocutor que tiene dificultades para pedir una ginebra al obtuso camarero. De este Interlocutor no oiremos nada, aunque, dato en absoluto irrelevante, iremos entreviendo algo de su actitud por las veces que el narrador le responde o le interpela. Al parecer, lo primero que sabemos es que muestra cierto interés por la historia que se le cuenta, que es poco más o menos la de una caída. En efecto, años atrás, en París, Clamence había sido un abogado de éxito. Armado de una contundente elocuencia, ejercía de defensor de las causas justas contra los abusos de la justicia. Correctamente disidente y perfecto en el punto de cortesía y atención a los desvalidos en nombre de un humanismo biempensante. Pero un acontecimiento inesperado, una suicida que se lanza al Sena sin que él mueva un dedo, desencadena un proceso de introspección que le va hundiendo poco a poco. Primero, es una especie de risa que viene

5 Sartre, Jean-Paul: "Réponse à Albert Camus”, en Situations, IV. Paris, Gallimard, 1964, p. 101.

6 Por ejemplo, "Défense de «L'Homme révolté»", OC III, pp. 366-378.

7 Aronson, Ronald: Camus y Sartre. La historia de una amistad y la disputa que le puso fin, Universidad de Granada, Universidad de Valencia, 2006, p. 282. 
de ninguna parte y que le acosa. Y a partir de ello, la rememoración de una serie de acontecimientos en los cuáles su papel no resultaba muy honroso. Descubre que sus buenas acciones tienen una causa oculta: su deseo de dominar, de estar por encima de las demás personas. Después, que la imagen pública, que constituye su instrumento de dominación, es vulnerable. Y finalmente, que la imagen de sí mismo como dominador se desmorona. Y todo esto es el motivo de su caída, que se produce no sólo por el sentimiento de culpabilidad, sino por la inseguridad que descubre al darse cuenta de que no está a la altura que se creía. Propiamente hablando, por la vergüenza.

Hasta aquí, y eso es lo que parece atraer al Interlocutor, la narración parece ser un acto de confesión. Pero, como ocurre en otros antecedentes -como Rousseau-, tras la exposición de todos los vicios y miserias propias se esconde una estrategia de dominación. Él mismo la explica al Interlocutor. No se trata de una confesión "grosera", sino un sutil autorretrato con los "rasgos comunes, las experiencias que hemos sufrido, las debilidades que compartimos, el buen tono, el hombre del día, en fin, todo lo que hace estragos en mí y en los otros". Cuando la requisitoria ha acabado, "el retrato que ofrezco a mis contemporáneos se convierte en un espejo" $"$.

Si la estrategia de Rousseau consiste en colocarse por encima de su auditorio en nombre de su absoluta sinceridad ${ }^{9}$, la de Clamence parte del supuesto de la indignidad compartida. Cuando descubre en sí mismo la hipocresía, la voluntad de dominación y la crueldad, la atribuye a la totalidad de la humanidad. "Cada hombre necesita esclavos como necesita el aire puro"10. Pero esto no tiene nada que ver con la sinceridad, sino con la formación de una máscara que funciona como un reflejo de quien la mira. A Clamence le interesa bien poco la verdad de los hechos de los que se acusa. Él elabora una narrativa, tal vez ficticia, que fuerza al Interlocutor a verse a sí mismo desde el punto de vista de la vergüenza.

Clamence llama "juez-penitente" a su nuevo rol. Sus presupuestos son claramente similares a los del sacerdote judeo-cristiano en la obra de Nietzsche ${ }^{11}$. Existe el punto común del resentimiento contra la vida, la represión del placer de la dominación y su exteriorización. También el sacerdote judeo-cristiano elabora una estrategia de dominación a través de la auto-humillación y la imputación universal de indignidad. Así se produce un vaivén entre la introyección y la externalización de la violencia, que lo mismo se reprime que se canaliza por cauces más efectivos y, sobre todo, más anti-vitales.

De manera similar, el juez-penitente se acusa. Confiesa todos los pecados, pero es en nombre del orgullo del dominante. Orientando su confesión en dirección de su culpa, tapa su vergüenza, que es mucho peor porque entraña su debilidad inconfesa. Y, ocultando ésta, la debilidad, consigue rebajar la humanidad del interlocutor y colocarse por encima, en calidad de develador de la infamia humana y profeta de sus consecuencias futuras.

8 La Chute, OC III, p. 761. A partir de ahora, $L C$.

9 "...que cada cual descubra luego su corazón, con sinceridad idéntica a la mía, a ver si hay alguno que se atreva entonces a decirte: Yo fui mejor que ese hombre" (Rousseau, 1979, 27).

10 LC, p. 716.

11 Deleuze, Gilles: Nietzsche et la philosophie, Paris, Presses Universitaires de France, 1967, p. 150. 


\section{Ginebra y fiebre}

Las referencias al alcohol son constantes en toda la obra. Ya de entrada, Clamence contacta con su potencial prosélito en torno a un vaso de ginebra. La bebida produce algunos efectos relajantes relacionados con el bienestar y la disipación del absurdo. En la vida nocturna de Paris, "el baile, el alcohol ligero, mi desenfreno" producían una fatiga durante la cual, por fracción de un segundo uno creía captar el sentido de la existencia ${ }^{12}$. Pero, obviamente, esta imprecisa sensación desaparecía junto con la laxitud. En realidad, la bebida está ligada en la novela con la enfermedad desde casi el principio. El alcohol y el sexo, llevados a su paroxismo, producen un sentimiento de "liberación" que, sin embargo, es destructivo. Cierto, el libertinaje al que se entrega Clamence como modo de vida anula todo sentido de la responsabilidad y de ligazón con el mundo y los otros, pero el cuerpo, "el hígado", le pone fin ${ }^{13}$. La enfermedad devuelve al ensoñador a la realidad y acaba, ya en Amsterdam, por instalarse de manera permanente o, al menos, recurrente. Pero, ¿qué enfermedad? No parece tratarse de ninguna en concreto. Clamence habla de ella en términos de fiebres, delirios, fatigas... "el paludismo, creo". Esta sintomatología es tan vaga que se puede sospechar si realmente hablamos de una dolencia biológica o de una enfermedad del alma. Desfallecimiento, estado febril... parecen apuntar a una especie de melancolía. Mucho más, cuando nos enteramos de que se "trata con ginebra" 14 , la misma bebida que es como una "luz dorada", especie de energía vital, que le permite caminar ensoñadoramente en las noches de Amsterdam ${ }^{15}$.

Obviamente, la bebida fue un sustituto de la fuerza vital en la etapa de Paris y seguirá siéndolo en el cuchitril de Clamence en Amsterdam, en la última jornada de su confesión. A nadie se le escapa las connotaciones de "refugiarse en la bebida" como falsa salida. Con ginebra o sin ella, la enfermedad acaba apoderándose del juez-penitente.

\section{La debilidad}

El tono de Clamence en las páginas finales de la obra, cambia sustancialmente respecto de las anteriores. Desde la primera escena, el contacto con el Interlocutor en el bar de Amsterdam, el flujo de su verborrea esconde una serie de pasos cuidadosamente medidos para provocar por sorpresa el efecto final. Las elipsis narrativas están calculadas para reforzar la tensión del relato y la curiosidad del interlocutor; la autoironía parece desviar la atención hacia el narrador, pero las sucesivas aproximaciones, en términos de aparentemente inocua complicidad, tienden a producir un efecto de identificación con él. La fuerza de Clamence reside en la retórica de las palabras y en su propia autoconfianza. Está tan seguro de sí mismo que no tiene inconveniente en desvelar las claves de su estrategia destructiva cuando calcula que el Interlocutor está suficientemente enredado en la malla. No hay que tener demasiado en cuenta la veracidad de los hechos que narra, dice. Quizás la historia de su "papado" en un campo de concentración, o la de sus amores brutales no sean ciertas.

12 LC, p. 710.

13 Ibid, p. 745.

14 Ibid, p. 751.

15 Ibíd, p. 701. 
Pero eso no importa. Los hechos narrados pueden ser falsos, pero significan una verdad de orden más profundo que cada cual debe buscar dentro de sí: la de la iniquidad en forma de hipocresía y la violencia de la dominación. “¿Las mentiras no llevan finalmente al camino de la verdad?"16 Hay, pues, algo cierto en lo que dice el falso profeta, aunque no se establezca en el terreno de los eventos, de los hechos.

Así pues, la condición de que el acto de confesión de Clamence sea un modelo insoslayable reside en la habilidad con que se exponga y la firmeza que aparente. La índole de la verdad que se promete no reside en ninguna metodología susceptible de contrastación, sino en la autoridad del enunciante. Así ha sido en toda la novela, hasta que el edificio se derrumba en el momento crítico, en la última jornada de la entrevista.

Ese día, Clamence comienza por verse obligado a recibir al Interlocutor en su habitación -escueta, pero pulcra-, enfermo, es decir, encamado. Y se siente confuso por ello. No puede ser de otra manera. Durante toda su confesión, ha jugado con la metáfora arriba-abajo, definiéndose como hombre de las alturas que trata de conseguir una posición superior a toda costa. “¿Cómo podría quedarme tranquilamente acostado? Me es preciso estar más alto que usted", afirma ahora ${ }^{17}$. En las jornadas anteriores ha ocupado el papel dominante en tanto que auxiliador, intermediario imprescindible, conductor del viaje por los caminos y las aguas de Amsterdam. La incesante actividad verbal revelaba una actividad dirigente subyacente que se efectuaba en el plano lingüístico, pero también conductual. Ahora que esta segunda, la actividad física, le resulta imposible, se encuentra literalmente rebajado. Es más, cuando en un momento dado intenta levantarse, delirante, para acudir a contemplar la nieve desde la ventana, es el Interlocutor el que se lo impide y, con ello, adopta una actitud dominante. Y esto resulta realmente insólito en el desarrollo del relato. "Ya me acuesto, perdóneme" (...) "Bueno, bueno, me tranquilizo, no se inquiete"18. Insólito. Por primera vez Clamence obedece a la voz que no oímos, pero que indudablemente nos representa en algún modo ${ }^{19}$.

Pero, más todavía, a pesar de su confianza en que le queda el poder de la palabra, también éste le abandona en un momento dado. Todo el esfuerzo del juez-penitente está encaminado a eliminar las carcajadas, metáforas de la vergüenza en la novela. Las risas que le acosan desde ninguna parte son la manifestación simbólica de la pérdida del poder de dominación mediante su exposición al juicio de la opinión pública, esto es, al ridículo. Después de diversos ensayos fallidos, la estrategia de la confesión provee al juez-penitente de un refugio seguro contra la vergüenza y de una nueva posición de poder. Las risas se han acabado. Pero, de pronto, el Interlocutor se ríe de él. Se ríe directamente cuando Clamence le invita a pasar a la confesión propia. “¡No se ría!”, grita. "Es usted un cliente difícil”20. El Interlocutor exaspera especialmente a Clamence no sólo porque se niega a entrar en el juego,

16 Ibíd, p. 752.

17 Ibid, p. 763.

18 Ibíd, p. 763.

19 Sobre el papel del Interlocutor, puede consultarse Cambier, Agnès: "Le quasi-soliloque de Janus", Revue d'histoire littéraire de la France, 4/2013 (Vol. 113), pp. 869-878. Consultado en línea, 13/05/2016 16:42, www.cairn.info/revue-d-histoire-litteraire-de-la-france-2013-4-page-869.htm. Se observará, sin embargo, que mi propuesta de análisis pretende ir más allá de la función narrativa del Interlocutor.

20 LC, p. 762. 
sino porque expresa esta negativa con el típico estímulo desencadenante de la vergüenza, la risa, manifestación de conmiseración o desprecio. Y para remachar el fracaso, cuando quiere reafirmar su estrategia cara al auditorio, el Interlocutor manifiesta su escepticismo. "¡Soy feliz, le digo que soy feliz, le prohíbo no creer que soy feliz, soy feliz a rabiar!”21, grita Clamence.

La razón del fracaso de Clamence, claramente expresado en estos dos momentos, se encuentra en la enfermedad. En las últimas páginas de la novela el protagonista se manifiesta continuamente en un estado febril y alterado. No sólo es que grite, como hemos visto en el párrafo anterior, sino que expresa un estado de vacilación y confusión que, por mucho que él pretenda que es controlada, resulta en una imagen infantilizada, carente de autodominio y potencia. Especialmente reveladoras son sus vacilaciones en cuanto a los símbolos que percibe. La confusión entre la nieve y las palomas, la rememoración visionaria de las islas perdidas del Pacífico, el entrelazamiento inextricable de los niveles diferentes de lo simbólico y lo real, marcan la pérdida de la realidad y la definición precisa, que eran la marca distintiva del espíritu de dominación de las jornadas anteriores.

\section{Jean-Baptiste y Albert: ¿Confesión o denuncia?}

He dicho más arriba "el auditorio" y he relacionado al Interlocutor con el lector/espectador varias veces. Al hacerlo, acepto el reto de Clamence cuando pasa del "yo" al "nosotros".

Estoy plantado delante de la humanidad, recapitulando mis vergüenzas, sin perder de vista el efecto que produzco, y diciendo "Yo era el último de los últimos". Entonces, insensiblemente, paso del "yo" al "nosotros". Cuando llego al "he aquí lo que somos", el juego está hecho, puedo decirles sus verdades ${ }^{22}$.

El mismo Camus calificó este juego como una representación escénica. "Utilicé aquí una técnica teatral (el monólogo dramático y el diálogo implícito) para describir un comediante trágico" ${ }^{23}$. Y, efectivamente, el relato acaba convirtiéndose en una dramática interpelación al espectador/lector. Voyeur cobijado tras el efecto de "cuarta pared", el lector se siente seguro, pero al pasar el discurso del "yo" al "nosotros", la mampara virtual desaparece de una manera súbita y se encuentra con la mirada del narrador que, por encima de los hombros del Interlocutor, exige de él un acto de introspección a la altura del que estaba observando despreocupadamente hasta ahora. Y no puede seguir juzgando. Es juzgado por su propia conciencia.

Si la cosa hubiera quedado aquí, hubiera significado el triunfo del juez-penitente y su cinismo nihilista. El triunfo de la no-vida en la ciudad muerta. Con ello Camus hubiera estado certificando su propia derrota frente a aquello que había estado combatiendo los

21 "Hereux à mourir" en el original. La expresión tiene su fuerza paradójica. La felicidad y la muerte juntas. He tomado "a rabiar" de la traducción de Alberto Luis Bixio (Camus, Albert: La caída, Buenos Aires, Losada, $3^{\mathrm{a}}$ edición, 1960) que conserva lo paradójico, aunque elimina el choque frontal de conceptos. Más no puede hacerse.

22 LC, p. 761.

23 En su última entrevista, publicada en el verano de 1960. (OC IV, p. 663). 
últimos años. ¿Sería el triunfo de sus críticos más ácidos el reconocerse como falso profeta? Hay quién lo ha sugerido. Pero hay que fijarse en que él introduce un mecanismo de defensa del lector, que no lo va a dejar abandonado frente a la poderosa retórica de Clamence: la enfermedad y el papel del Interlocutor.

Primero, la enfermedad. El procedimiento de Clamence es un juego de potencias. Aparentando ser el último de los hombres, trata de asumir el rol de conductor de conciencias hacia la aniquilación de lo humano. Y le es posible porque se muestra seguro de sí. $\mathrm{Si}$, como hemos visto, la enfermedad desmonta esta seguridad, su poder de dominación desaparece. Porque el juez-penitente es un intelectual. No tiene más mecanismo de poder que su palabra.

Y luego, el Interlocutor, que duda de su palabra y se resiste con su risa, demuestra que Clamence ha perdido su poderío. Este rol es de gran importancia, aunque con frecuencia se pase por alto. Es cierto, como dice Fitch ${ }^{24}$, que el discurso de Clamence se dirige al lector por encima del Interlocutor, como dije antes, pero éste no es un recurso significativamente "insubstancial". Porque no es pasivo, actúa. Y su acción es claramente significativa: una resistencia. Los resultados de esta resistencia son inciertos, tal como se refleja en la novela, pero, independientemente de ello, aquí se marca un camino de oposición al nihilismo, que fue el hilo conductor de la actividad literaria y política de Camus hasta su misma muerte.

Según ello, en un primer nivel simbólico, la enfermedad es la debilidad del nihilista. Una enfermedad que hace confuso su discurso y que, sobre todo, revela su falta de contacto con la realidad, que en el concepto camusiano corresponde a la vida. El diagnóstico y la prescripción estaban ya establecidos en El hombre rebelde. Allí el nihilista se caracteriza por crear un universo abstracto de sumisión y dominación alejado de la vida. Abstracta es su prédica del futuro ideal, abstractos sus sistemas de totalización de la realidad, abstracta su justificación del asesinato político. "Cuando la idea de inocencia desaparece en el mismo inocente, el valor del poder reina definitivamente en un mundo desesperado. Es por eso que la cruel penitencia reina en un mundo donde sólo las piedras son inocentes" 25 . La posterior querella con Jeanson y Sartre no hace sino poner nombres al mal que se señalaba entonces. "Temps modernes ${ }^{26}$. Admiten el pecado y rehúsan la gracia. Sed de martirio"27. "Existencialismo. Cuando se acusan es para abrumar a los otros. Jueces penitentes"28. En 1953 anota en sus Carnets: "El impromptu de los filósofos en comedia dell'arte"29. Lo que

24 Fitch, Brian T.: The Fall. A Matter of Guilt, New York, Twayne, 1995, p. 117. Sobre el mismo tema, pero dando sustancialidad al Interlocutor, cf. Peuchet,Sylvie: «L'interlocuteur », en L. Arsac et al. Analyses et réflexions sur Albert Camus, La Chute. Paris, Ellipses, 1997, pp. 56ss y Abbou, André: Albert Camus, entre les lignes Adieu à la littérature ou fausse sortie ? 1955-1959, Paris, Éditions Séguier, 2009, pp. 259-60.

25 L'Homme révolté, OC III, p. 219.

26 En cursiva en el original. Referencia a la revista dirigida por Sartre en la que tuvo lugar el enfrentamiento de 1952.

27 Carnets, OC IV, p. 1144.

28 Ibíd, p. 1212.

29 Ibíd,p. 1173. Con el nombre de L'Impromptu des philosophes y el seudónimo de Antoine Bailly, Camus guardó entre sus papeles una comedia satírica escrita casi con seguridad en 1947 que no será publicada hasta 2006. El protagonista, una especie de tartufo embrollón y vacuo, responde al significativo nombre de Sr. Nada. Parece que en 1953 Camus consideraba llevarla a escena. 
se añade ahora es un análisis de orden psicológico y político: el placer del expediente de la sumisión que se oculta tras la cháchara de las buenas intenciones. No cabe duda, pues, de que con Clamence Camus está haciendo un retrato psico-político de Jean-Paul Sartre. Simone de Beauvoir no dejó de verlo cuando escribe que la primera parte de La Caída es una gran obra en la que se recupera el mejor Camus, pero que en la segunda se deja llevar por "el resentimiento" 30 . Léase con "resentimiento" el ataque frontal contra Sartre. Esta es la primera lectura.

Pero aunque el propio Camus enfatice que lo importante de su universo simbólico son las primeras interpretaciones (lo demás se debería al azar), hay elementos suficientes para analizar más en profundidad el juego de los significados "secundarios", si se quiere decir así, en las andanzas de Jean-Baptiste Clamence y, más en concreto, en el rol de la enfermedad. Desde el primer momento, se advirtió que el personaje del juez-penitente compartía con el propio Camus un amplio abanico de hechos y dichos que parecía sobrepasar la pura casualidad. Sus amigos y sus enemigos más íntimos estaban convencidos de que a través de los significados obvios de la obra -el ataque a "nuestros existencialistas"- se transparentaba algo así como una autocrítica feroz ${ }^{31}$. Camus lo niega una y otra vez, pero la sospecha tiene sus bases. La certeza es otra cosa.

En todo caso, ciñéndonos al asunto que nos ocupa, hay que hacer una constatación evidente: independientemente de qué o de quién hablemos, la enfermedad tal como Camus la muestra en La caída, es una derrota. En la novela se aplica a la voluntad de dominación, pero sus efectos devastadores (pérdida de la realidad, confusión, actos divagantes, tensión del vivir para la muerte) podrían entenderse como contradicción de cualquier proyecto existencial que pretenda basarse en la lucidez y en la exaltación de la vida, como era justamente el caso de Camus. Las crisis personales, vinculadas en muchos casos a rebrotes de la tuberculosis (la dificultad de respirar de Clamence), han dejado una serie de anotaciones en este sentido que permiten seguramente una indagación más minuciosa de lo que estas líneas permiten. Queda, pues, la cuestión abierta.

\section{Bibliografía}

Abbou, André (2009): Albert Camus, entre les lignes - Adieu à la littérature ou fausse sortie? 1955-1959, Paris, Éditions Séguier.

Aronson, Ronald (2006): Camus y Sartre. La historia de una amistad y la disputa que le puso fin., Universidad de Granada, Universidad de Valencia.

Beauvoir, Simone de (1964): La fuerza de las cosas. Buenos Aires, EDHASA.

Cambier, Agnès (2013): "Le quasi-soliloque de Janus", Revue d'histoire littéraire de la France, 4/2013 (Vol. 113), pp. 869-878. Consultado en línea, 13/05/2016 16:42 www. cairn.info/revue-d-histoire-litteraire-de-la-france-2013-4-page-869.htm.

Camus, Albert (1960): La Caída, trad. de Alberto Luís Bixio, Buenos Aires, Losada, 1960

Camus, Albert (2006): CEuvres complètes. 4 Vol., J. Lévi-Valensi y R. Gay-Crosier eds., Paris, Gallimard, Coll., de la Pléiade.

30 Beauvoir, Simone de: La fuerza de las cosas. Buenos Aires, EDHASA, 1964, p. 412.

31 Todd, op. cit., p. 640. 
Deleuze, Gilles (1967): Nietzsche et la philosophie, Paris, Presses Universitaires de France. Fitch, Brian T. (1995): The Fall. A Matter of Guilt, New York, Twayne.

Jeanson, Francis (1964): “Albert Camus o el alma rebelde”, en Sartre, Jean-Paul; Camus, Albert; Jeanson, Francis: Polémica Sartre-Camus, Buenos Aires, El Escarabajo de Oro. Peuchet, Sylvie (1997): “L'Interlocuteur", en L. Arsac et al., Analyses et réflexions sur Albert Camus, La Chute, Paris, Ellipses, pp. 56-60.

Rousseau, Juan Jacobo (1979): Las confesiones, Madrid, Espasa Calpe.

Sartre, Jean-Paul (1964): "Réponse à Albert Camus", en Situations, IV. Paris, Gallimard, pp. 90-125.

Todd, Oliver (1997): Albert Camus. Una vida, Barcelona, Tusquets. 